\title{
Targeting glutamate to treat schizophrenia: lessons from recent clinical studies
}

\author{
Katherine Beck $^{1}$ • Daniel C Javitt ${ }^{2,3}$ - Oliver D Howes ${ }^{1,4}$
}

Received: 28 February 2016 / Accepted: 3 March 2016 / Published online: 23 May 2016

(C) Springer-Verlag Berlin Heidelberg 2016

On the face of it, the treatment of schizophrenia offers lots of choice: there are more than 30 antipsychotic drugs licensed for first-line treatment. However, as they all essentially use the same common mechanism of action, D2 antagonism, from a mechanistic perspective, there is little choice (Howes and Kapur 2009). This is a problem because one third of patients do not respond to standard medications (Beck et al. 2014), and the drugs have limited efficacy for negative and cognitive symptoms. This is not surprising as both treatment resistance and negative and cognitive symptoms seem to involve other neurotransmitter systems (Mouchlianitis et al. 2015). Therefore, there is a need to develop novel treatments targeting other neurotransmitters.

Emerging evidence suggests a key role for glutamate dysfunction in the pathophysiology of schizophrenia-in particular, $N$-methyl-D-aspartate-receptor (NMDAR) hypofunction (Javitt and Zukin 1991; Coyle 1996). NMDAR antagonists are able to mimic the full range of symptoms seen in schizophrenia (Krystal et al. 1994), and genetic, post mortem and animal studies lend further support to the NMDAR

Katherine Beck

katherine.beck@kcl.ac.uk

$\checkmark$ Oliver D Howes

oliver.howes@kcl.ac.uk

1 Department of Psychosis Studies, Institute of Psychiatry, Psychology and Neuroscience, King's College London, London, UK

2 Division of Experimental Therapeutics, Departments of Psychiatry and Neuroscience, Columbia University Medical Center, 1051 Riverside Drive, New York, NY 10032, USA

3 Schizophrenia Research Division, Nathan Kline Institute for Psychiatric Research, 140 Old Orangeburg Road, Orangeburg, NY 10962, USA

4 Psychiatric Imaging Group, Imperial College, MRC Clinical Sciences Centre, Hammersmith Hospital, London, UK hypothesis. This has led to a drive to develop drugs targeting the NMDAR. One approach has been to target the NMDAR's glycine binding site. Glycine is required to bind at the same time as glutamate to allow the activation of the NMDAR (Kleckner and Dingledine 1988). Increasing glycine occupancy has the potential to increase NMDAR neurotransmission (Danysz and Parsons 1998).

Early clinical trials conducted at single sites found that glycine administered in combination with antipsychotics could reduce negative and cognitive symptoms when compared with antipsychotics alone (Javitt et al. 1994; HerescoLevy et al. 1996, 1999, 2004). However, a multi-centre study did not show separation from placebo (Buchanan et al. 2007). Furthermore, glycine does not easily cross the blood-brain barrier, necessitating high doses, affecting its tolerability. Alternative approaches to increasing brain glycine have been sought. Of particular interest is the glycine transporter 1 (GlyT1), which is postulated to be a key regular of synaptic glycine (Supplisson and Bergman 1997).

A number of GlyT1 inhibitors have been synthesised and trialled. However, only the Hoffmann-La Roche compound, bitopertin, has reached phase III clinical trials (Singer et al. 2015). Bitopertin is a selective and potent glycine reuptake inhibitor (GRI). A phase II proof of concept study, of patients with schizophrenia with predominant negative symptoms, found that bitopertin (at doses of 10 and $30 \mathrm{mg}$ daily) moderately reduced negative symptoms (Umbricht et al. 2014). However, this was not significant in the intention-to-treat analysis.

A clinical trial programme called "SearchLyte" was instigated. It consisted of six phase III studies. Three of these targeted the drug's ability to treat patients with persistent negative symptoms. The other three studies were designed to determine the drug's ability to reduce positive symptoms in patients who had not responded to the antipsychotic drugs currently available. Two of the trials were discontinued after 
a futility analysis (Singer et al. 2015). The remaining trials did not meet their primary endpoints with the exception of the NightLyte study. This included patients with a suboptimal response to previous antipsychotics and found that the addition of bitopertin $10 \mathrm{mg}$ significantly improved the Positive and Negative Syndrome Scale (PANSS) positive factor score.

Whilst the phase III trials focussed on the adjunctive use of bitopertin, Roche also completed a phase II/III monotherapy trial (Bugarski-Kirola et al. 2014). This included patients with an acute exacerbation of illness, and comparisons with both a placebo and an active control group, using olanzapine. It found a trend for improvement in the total PANSS, which was the study's primary endpoint, for both bitopertin $(30 \mathrm{mg})$ and olanzapine. However, this did not significantly differ from placebo for either drug. As a result, it was considered a failed study. Nevertheless, bitopertin showed a significant improvement in the PANSS positive subscale score and an increase in readiness for hospital discharge compared to placebo. Unfortunately, bitopertin is no longer being developed for use as an antipsychotic.

Similar problems occurred in the development of mGluR2/3 agonists, which act by inhibiting glutamate release ( $\mathrm{Li}$ et al. 2015). Despite a promising proof of concept study finding improved positive and negative symptoms after treatment with LY2140023 compared with placebo (Patil et al. 2007), later trials were unsuccessful. One follow-up multi-centre phase II trial was inconclusive, with a high placebo response rate (Kinon et al. 2011), and another phase II open-label study found LY2140023 was inferior to a comparison atypical antipsychotic ( $\mathrm{Li}$ et al. 2015). In August 2012, Eli Lilly announced their decision to stop phase III trials as one of the trials, closest to completion, failed to meet its primary endpoint ( $\mathrm{Li}$ et al. 2015).

\section{What could underlie these disappointing findings?}

The first possibility, of course, is that CNS levels of the drugs were inadequate and/or variable between patients, and, in the case of bitopertin, levels of glycine consequently inadequate for therapeutic effects. In this issue of Psychopharmacology, Hofmann et al. 2016 measure plasma and CNS levels of bitopertin and another glycine transporter inhibitor (RG7118). They find that bitopertin and RG7118 both exhibit a dose-dependent linear relationship between plasma and cerebrospinal fluid glycine concentrations in healthy controls. This study confirms bitopertin's ability to enter the CNS and increase glycine levels. Moreover, the variability between subjects is relatively low, at least for the 30- and 60-mg doses. As there is no reason to think that patients with schizophrenia will be different in this respect, the Hofmann et al. 2016 findings seem to rule out failure of bitopertin to reliably enter the
CNS and increase glycine levels as an explanation for the inconsistent findings.

What then could explain the disappointing trial results with bitopertin? One possibility is the effect of placebo response. In contrast to Umbricht's phase II study, the phase III studies had much stronger placebo responses, which make it harder to show a treatment effect. Unusually high placebo responses were also found in clinical trials of LY2140023 (Li et al. 2015). Placebo responses are a particular concern for studies of negative symptoms, as these may be both primary, or secondary. Primary negative symptoms are intrinsic to the illness, and thus potentially responsive to interventions targeting the underlying biology, whilst secondary negative symptoms are due to other factors such as social isolation and stigma. The latter are likely to be responsive to psychosocial intervention and so may improve with the regular social interactions seen as part of the assessments and medical reviews in a clinical trial, contributing to a large placebo effect. The phase III studies were in chronic patients, who are likely to have developed secondary negative symptoms. Future studies should focus on patients early in the course of the illness, where negative symptoms will be predominantly primary. Another problem with the inclusion of chronic patients in the phase III studies is that there is evidence that glutamate abnormalities are more prominent early in the course of the illness (Poels et al. 2014), and drugs targeting this system may only have an effect in patients in early stages of illness.

At present there is a "disconnect" in the literature between the high reported effectiveness of sarcosine, a naturally occurring, non-specific glycine transport inhibitor, vs. the more modest effectiveness of highly selective GlyT1 inhibitors such as bitopertin. Although GlyT1 transporters have the highest specificity in the brain for glycine, other glycine transporters are also present and may be more relevant than GlyT1 for control of synaptic glycine levels. For example, the small neutral amino acid transport (SNAT, SLC38) system, also termed System A, appears to play a more specific role in the regulation of synaptosomal glycine transport than GlyT1 and also may contribute to the unique therapeutic efficacy of clozapine (Javitt et al. 2005). Future studies with less-selective glycine transport inhibitors may therefore be warranted.

Finally, clinical trials tend to ignore the fact that schizophrenia is a highly heterogeneous disorder and it is unlikely that one medication will treat all patients (Howes and Kapur 2014). Roche took the approach of stratifying by clinical phenotype, but the possibility of phenocopies mean this is unreliable. What is needed is stratification by underlying biology. Genome-wide association studies recently identified a potential association between schizophrenia and variants at GRIN2A (the gene for the NR2A subunit of the NMDAR) (Lencz and Malhotra 2015), and studies using proton magnetic resonance spectroscopy (MRS) have found that patients who do not respond to conventional medications targeting 
the dopamine system have elevated glutamate levels compared with responders (Egerton et al. 2012; Demjaha et al. 2014; Mouchlianitis et al. 2015). Thus, trials of glutamate targeting drugs could recruit patients with these genetic risk variants and/or glutamate abnormalities on imaging assessment. Furthermore, neuroimaging biomarkers could then be used to assess drug effects. This would mean that a negative trial would be much more informative to the field, as it would show whether the drug targeted the relevant system or not. The Hofmann et al. 2016 study illustrates the value of measuring biomarkers. It would have been informative to include them in the patient studies as well.

Should we take the failure of bitopertin, and LY2140023, in phase III development as the death knell of glutamatergic approaches to treating schizophrenia? The emerging genetic and neuroimaging evidence suggests not. Instead, we need to become smarter in how we conduct clinical studies.

\section{Compliance with ethical standards}

Funding This study was funded by the Medical Research Council-UK (no. MC-A656-5QD30), Maudsley Charity (no. 666), Brain and Behavior Research Foundation, and Wellcome Trust (no. 094849/Z/10/ Z) grants to Dr Howes and the National Institute for Health Research (NIHR) Biomedical Research Centre at South London and Maudsley NHS Foundation Trust and King's College London.

Conflict of interest Dr Beck declares that she has no conflict of interest.

Dr. Javitt reports grants from the NIMH, Stanley Foundation and Roche. Personal fees from Takeda and Forum, outside the submitted work; in addition, Dr. Javitt has a patent for Glycine, D-serine in schizophrenia issued, a patent for Glycine transport inhibitors in schizophrenia issued, a patent D-serine in Movement Disorders issued, and a patent for D-cycloserine in depression pending. Dr. Javitt reports equity interest in Glytech and NeuroRx, and serves on the advisory board of Promentis.

Dr Howes has received investigator-initiated research funding from and/or participated in advisory/speaker meetings organised by AstraZeneca, Autifony, BMS, Eli Lilly, Heptares, Jansenn, Lundbeck, Lyden-Delta, Otsuka, Servier, Sunovion, Rand and Roche. Neither Dr Howes nor his family have been employed by or have holdings/a financial stake in any biomedical company.

\section{References}

Beck K, McCutcheon R, Bloomfield MA, Gaughran F, Reis Marques T, MacCabe J, Selvaraj S, Taylor D, Howes OD (2014) The practical management of refractory schizophrenia - the Maudsley Treatment REview and Assessment Team service approach. Acta Psychiatr Scand 130(6):427-438

Buchanan RW, Javitt DC, Marder SR, Schooler NR, Gold JM, McMahon RP, Heresco-Levy U, Carpenter WT (2007) The Cognitive and Negative Symptoms in Schizophrenia Trial (CONSIST): the efficacy of glutamatergic agents for negative symptoms and cognitive impairments. Am J Psychiatry 164(10):1593-1602

Bugarski-Kirola D, Wang A, Abi-Saab D, Blattler T (2014) A phase II/III trial of bitopertin monotherapy compared with placebo in patients with an acute exacerbation of schizophrenia - results from the CandleLyte study. Eur Neuropsychopharmacol 24(7):1024-1036

Coyle JT (1996) The glutamatergic dysfunction hypothesis for schizophrenia. Harv Rev Psychiatry 3(5):241-253

Danysz W, Parsons CG (1998) Glycine and N-methyl-D-aspartate receptors: physiological significance and possible therapeutic applications. Pharmacol Rev 50(4):597-664

Demjaha A, Egerton A, Murray RM, Kapur S, Howes OD, Stone JM, McGuire PK (2014) Antipsychotic treatment resistance in schizophrenia associated with elevated glutamate levels but normal dopamine function. Biol Psychiatry 75(5):e11-e13

Egerton A, Brugger S, Raffin M, Barker GJ, Lythgoe DJ, McGuire PK, Stone JM (2012) Anterior cingulate glutamate levels related to clinical status following treatment in first-episode schizophrenia. Neuropsychopharmacology 37(11):2515-2521

Heresco-Levy U, Javitt DC, Ermilov M, Mordel C, Horowitz A, Kelly D (1996) Double-blind, placebo-controlled, crossover trial of glycine adjuvant therapy for treatment-resistant schizophrenia. Br J Psychiatry 169(5):610-617

Heresco-Levy U, Javitt DC, Ermilov M, Mordel C, Silipo G, Lichtenstein M (1999) Efficacy of high-dose glycine in the treatment of enduring negative symptoms of schizophrenia. Arch Gen Psychiatry 56(1): 29-36

Heresco-Levy U, Ermilov M, Lichtenberg P, Bar G, Javitt DC (2004) High-dose glycine added to olanzapine and risperidone for the treatment of schizophrenia. Biol Psychiatry 55(2):165-171

Hofmann C, Pizzagalli F, Boetsch C, Alberati D, Ereshefsky L, Jhee S, Patat A, Boutouyrie-Dumont B, Martin-Facklam M (2016) Effects of the glycine reuptake inbitors bitopertin and RG7118 on glycine in cerebrospinal fluid: results of two proofs of mechanism studies in healthy volunteers. Psychopharmacology. doi: 10.1007/s00213016-4317-7

Howes OD, Kapur S (2009) The dopamine hypothesis of schizophrenia: version III - the final common pathway. Schizophr Bull 35(3):549 562

Howes OD, Kapur S (2014) A neurobiological hypothesis for the classification of schizophrenia: type A (hyperdopaminergic) and type B (normodopaminergic). Br J Psychiatry 205(1):1-3

Javitt DC, Zukin SR (1991) Recent advances in the phencyclidine model of schizophrenia. Am J Psychiatry 148(10):1301-1308

Javitt DC, Zylberman I, Zukin SR, Heresco-Levy U, Lindenmayer JP (1994) Amelioration of negative symptoms in schizophrenia by glycine. Am J Psychiatry 151(8):1234-1236

Javitt DC, Duncan L, Balla A, Sershen H (2005) Inhibition of system Amediated glycine transport in cortical synaptosomes by therapeutic concentrations of clozapine: implications for mechanisms of action. Mol Psychiatry 10(3):275-287

Kinon, B. J., L. Zhang, B. A. Millen, O. O. Osuntokun, J. E. Williams, S. Kollack-Walker, K. Jackson, L. Kryzhanovskaya, N. Jarkova and H. S. Group (2011) A multicenter, inpatient, phase 2, double-blind, placebo-controlled dose-ranging study of LY2140023 monohydrate in patients with DSM-IV schizophrenia. J Clin Psychopharmacol 31(3):349-355

Kleckner NW, Dingledine R (1988) Requirement for glycine in activation of NMDA-receptors expressed in Xenopus oocytes. Science 241(4867):835-837

Krystal JH, Karper LP, Seibyl JP, Freeman GK, Delaney R, Bremner JD, Heninger GR, Bowers MB Jr, Charney DS (1994) Subanesthetic effects of the noncompetitive NMDA antagonist, ketamine, in humans. Psychotomimetic, perceptual, cognitive, and neuroendocrine responses. Arch Gen Psychiatry 51(3):199-214

Lencz T, Malhotra AK (2015) Targeting the schizophrenia genome: a fast track strategy from GWAS to clinic. Mol Psychiatry 20(7):820-826

Li ML, Hu XQ, Li F, Gao WJ (2015) Perspectives on the mGluR2/3 agonists as a therapeutic target for schizophrenia: still promising or a dead end? Prog Neuropsychopharmacol Biol Psychiatry 60:66-76 
Mouchlianitis E, Bloomfield MA, Law V, Beck K, Selvaraj S, Rasquinha N, Waldman A, Turkheimer FE, Egerton A, Stone J, Howes OD (2015) Treatment-resistant schizophrenia patients show elevated anterior cingulate cortex glutamate compared to treatment-responsive. Bull, Schizophr

Patil ST, Zhang L, Martenyi F, Lowe SL, Jackson KA, Andreev BV, Avedisova AS, Bardenstein LM, Gurovich IY, Morozova MA, Mosolov SN, Neznanov NG, Reznik AM, Smulevich AB, Tochilov VA, Johnson BG, Monn JA, Schoepp DD (2007) Activation of mGlu2/3 receptors as a new approach to treat schizophrenia: a randomized phase 2 clinical trial. Nat Med 13(9):11021107

Poels EM, Kegeles LS, Kantrowitz JT, Slifstein M, Javitt DC, Lieberman JA, Abi-Dargham A, Girgis RR (2014) Imaging glutamate in schizophrenia: review of findings and implications for drug discovery. Mol Psychiatry 19(1):20-29

Singer P, Dubroqua S, Yee BK (2015) Inhibition of glycine transporter 1: the yellow brick road to new schizophrenia therapy? Curr Pharm Des 21(26):3771-3787

Supplisson S, Bergman C (1997) Control of NMDA receptor activation by a glycine transporter co-expressed in Xenopus oocytes. $\mathrm{J}$ Neurosci 17(12):4580-4590

Umbricht D, Alberati D, Martin-Facklam M, Borroni E, Youssef EA, Ostland M, Wallace TL, Knoflach F, Dorflinger E, Wettstein JG, Bausch A, Garibaldi G, Santarelli L (2014) Effect of bitopertin, a glycine reuptake inhibitor, on negative symptoms of schizophrenia: a randomized, double-blind, proof-of-concept study. JAMA Psychiatry 71(6):637-646 